\title{
COMPARISON BY SIMULATION OF SPEED CONTROLLERS OF THE SOCCER PLAYER ROBOT SYSTEM OF SSL CATEGORY PROJECT AGENTCOOP
}

\author{
COMPARACIÓN POR MEDIO DE LA SIMULACIÓN DE LOS \\ CONTROLADORES DE VELOCIDAD DEL SISTEMA DE ROBOT JUGADOR \\ DE FUTBOL CATEGORÍA SSL PROYECTO AGENTCOOP
}

\author{
MSc. Tania Liseth Acevedo Gauta, * \\ MSc. Andrés Orlando Paez Melo, ** \\ MSc. Milton Osvaldo Amarillo Rojas, *** \\ Universidad de Pamplona, * \\ Universidad de Pamplona, ** \\ Universidad Nacional Abierta y a Distancia UNAD, **** \\ tl_acevedo@hotmail.com, andres.paez2@unipamplona.edu.co, \\ milton.amarillo@unad.edu.co
}

\begin{abstract}
This project presents the simulation of speed controllers for each of the motors that control the wheels of a holonomic robot system, that was designed at the university Javeriana for the project Agencoop robots soccer players category SSL. The first simulation is based on the real controllers that have the robots of the project Agentcoop category SSL developed in the Pontifica Universidad Javeriana, the second simulation is a proposal to improve the response speed of the current project controller. Finally, the comparison of the two controllers that were simulated considering the establishment time like a performance parameter.
\end{abstract}

Keywords: Simulation, modeling, design, fuzzy controller, velocity, stability, PI control.

Resumen: En este proyecto, se presentan la simulación de dos controladores de velocidad para cada uno de los motores que controlan las ruedas de un sistema de robot holonómico, diseñado en la universidad Javeriana para el proyecto Agencoop robots jugadores de futbol categoría SSL. La primera simulación se basa en los controladores reales que tienen los robots del proyecto Agentcoop categoría SSL desarrollados en la Pontifica Universidad Javeriana, la segunda simulación es una propuesta para mejorar la velocidad de respuesta que tiene el controlador actual del proyecto. Finalmente se realiza la comparación de los dos controladores que se simularon teniendo en cuenta el tiempo de establecimiento como parámetro de rendimiento.

Palabras clave: Simulación, modelado, diseño, control difuso, velocidad, estabilidad control PI.

\section{INTRODUCIÓN}

Como en los partidos de futbol humanos, en los partidos de futbol robóticos se hace evidente que una mayor velocidad de los jugadores genera ventajas al momento de alcanzar el balón y jugar los partidos. Analizando las entradas de los motores de los robots del proyecto Agentcoop categoría SSL, se encuentra que para puntos de operación de velocidades bajas y medianas el controlador no utiliza toda la energía disponible para llegar lo más rápido posible a la velocidad deseada.

Para mejorar tiempo de respuesta que tienen actualmente motores de los robots del proyecto Agentcoop categoría SSL Valderrama (2013), en este trabajo se diseña un controlador alternativo por medio de simulación, que intente disminuir el tiempo de respuesta para puntos de operación de velocidades bajas y medianas. 
Si se busca mejorar la velocidad de respuesta de los controladores en puntos de operación de velocidades medianas y bajas, se debe tratar de utilizar la mayor cantidad de energía disponible para estos casos. Esto se puede logar utilizando una técnica de control no lineal que fuerce al sistema a entregar más energía en estos casos específicos. El control difuso además de ser una técnica no muy compleja reúne características de no linealidad que podemos utilizar para mejorar el tiempo de respuesta de los motores.

Teniendo en cuenta el trabajo de Ahmed (2016) encontramos que se obtienen mejores resultados utilizando un controlador difuso respecto al controlador PI aplicado en un rectificador de 3 niveles, hay que tener en cuenta que el sistema rectificador es un sistema no lineal, que tiene una dinámico de carga y descarga de un condensador (rizado de voltaje). En un principio se trató de implementar un diseñó similar al de Ahmed (2016), un control difuso que dependiera del error y la diferencia del error, obteniendo un error significativo de estado estacionario. Se investigaron otras alternativas para el diseño de controladores PI utilizando el control difuso y se encontró el trabajo de Jing (2016) donde se combina un control difuso y un control PI dependiendo del error, también se encontró el trabajo de Li (2017) done los parámetros del control PI variables por medio de un control difuso.

Teniendo en cuenta los trabajos encontrados y las limitaciones del procesador, se optó por encontrar dos controladores; uno difuso para puntos de operación de velocidades bajas y medianas que dependiera del error y la acumulación del error (integral) (Morales, 2009), $\mathrm{y}$ otro que funcione para entradas altas. En trabajo tiene un control similar al del trabajo de Jing (2016) pero donde se combinan dos controladores difusos que dependen del punto de operación.

\section{PROYECTO AGENTCOOP ROBOTS JUGADORES DE FUTBOL SSL}

Una de las principales ramas de estudio en la ingeniería de control es la robótica móvil, la cual hace uso de la automatización y la inteligencia artificial para crear dispositivos autónomos. Una de las aplicaciones con mayor acogida por es el campeonado de futbol de robots "Robocopa", en este campeonato hay una liga de robots de pequeño tamaño denomina SSL. Los grupos de investigación SIDRE y SIRP de la Pontificia Universidad Javeriana crearon un equipo con los correspondientes robots (Valderrama, 2013, p 5). Entre las reglas que establece la Robocopa sobre esta categoría está el tamaño, equipos de visión y marcadores (Noda, s.f.). Generalmente los robots son holonómicos, ósea que las llantas son omnidireccionales (puede moverse en cualquier sentido) como se muestra en la Figura 1, esto es para generar un menor desplazamiento hacia el balón independientemente del Angulo. Este diseño es para disminuir el tiempo de llegada hacia el balón, ya que si se llega primero al balón se tiene el dominio de este (genera ventaja para el equipo que primero llegue).

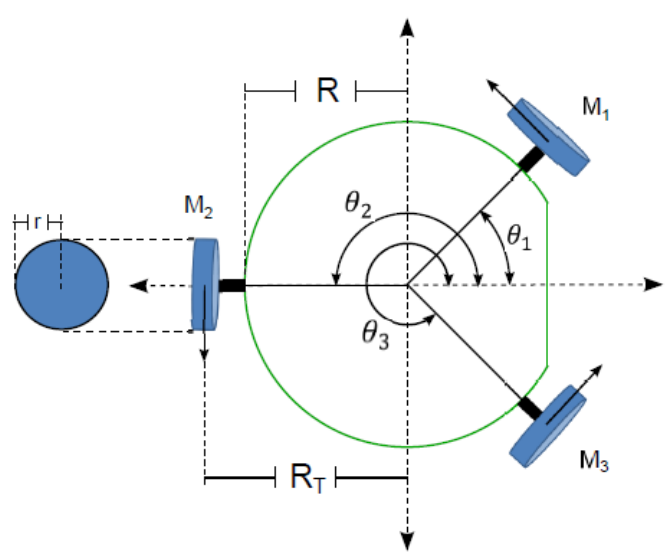

Fig. 1. Estructura del robot holonómico de liga SSL (Valderrama, 2013, p 25).

\subsection{Características de los robots}

El diseño general de los robots para jugar futbol en la Robocopa liga SSL se muestra en la figura 1. Los robots constan de 3 motores $\left(M_{1}, M_{2}\right.$ y $\mathrm{M}_{3}$ ) que mueven las ruedas separadas 180 grados cada una para el desplazamiento omnidireccional del robot. Cada uno de los motores recibe un valor de referencia de velocidad para realizar el desplazamiento.

Para llegar al valor deseado de velocidad de los motores y mantenerlos constantes se debe usar una estrategia de control.

\subsection{Modelado y control PI desarrollado en el proyecto Agencoop para los motores}

Para modelar cada motor de las ruedas teniendo en cuenta como entrada el voltaje y la salida la velocidad, la tesis de grado Valderrama (2013), utiliza un sistema de primer orden (Ogata, 2010):

$$
\frac{\operatorname{vel}(t)}{\operatorname{vol}(t)}=\frac{K}{\tau s+1}
$$

Donde $\tau=71 \mathrm{~ms}, \mathrm{y} \mathrm{K}=1,07$ V.m/t. En la Figura 2 , se encuentra la respuesta real de los motores para diferentes valores de la entrada.

Suponiendo un tiempo de muestreo de $10 \mathrm{~ms}$ según los cálculos del procesador y utilizando el 
método Zero Order Hold se obtiene el modelo en tiempo discreto (Valderrama, 2013):

$$
G(z)=\frac{0.1388}{z+0.8703}
$$

El motor se controla con entradas de $0 \%$ a $100 \%$ del voltaje máximo que tengan las baterías en ese instante (Valderrama, 2013).

Respuesta paso de dos Motores a Diferentes Valores

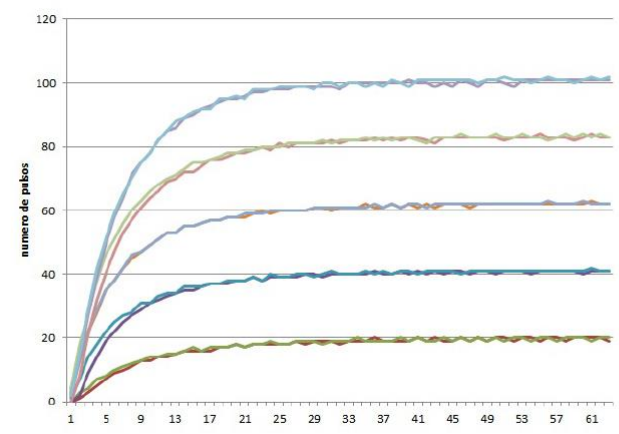

tiempo x 10 milisegund

Fig. 2. Respuesta de velocidad real de dos motores (Valderrama, 2013)

Como parte inicial del desarrollo se realiza la verificación de la planta identificada anteriormente en la tesis de grado Valderrama (2013). Se toman los datos del sistema de primer orden tanto en continuo como discreto y se aplica como entrada un escalón unitario. En la Figura 3 se muestra el diagrama de bloques de un motor con su respectiva entrada paso y en la Figura 4 se muestra la respuesta de la simulación que según la tesis de grado Valderrama (2013) concuerdan muy bien.

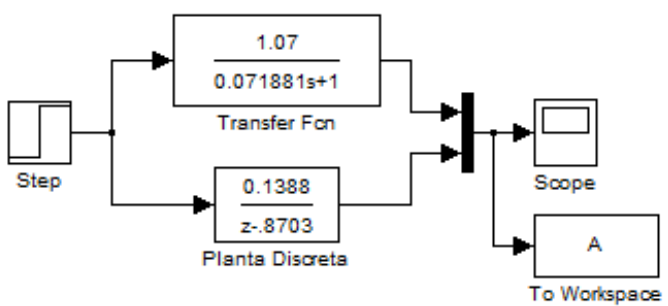

Fig. 3 Diagrama de bloques del modelo de cada motor en Matlab simulink®

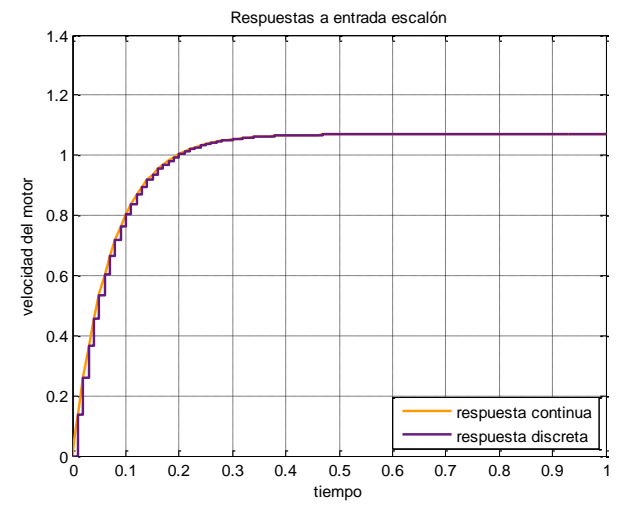

Fig. 4. Respuesta de velocidad del motor en tiempo continuo y discreto ante una entrada escalón unitario

Se obtienen las gráficas en correspondencia con los sistemas contínuo y discreto representados en los modelos matemáticos previamente modelados.

2.3 Simulación de un controlador alterno que mejore el tiempo en respuesta de velocidad de los motores

El controlador PI desarrollado en la tesis de grado Valderrama (2013), se diseñó con base en que la respuesta paso no tuviera sobre pico, las constantes encontradas fueron $\mathrm{K}_{\mathrm{P}}=1.75 \mathrm{y}$ $\mathrm{K}_{\mathrm{I}}=26.9$, la comparación entre el control real aplicado y la simulación obtenido por Valderrama (2013), se puede observar en la Figura 5.

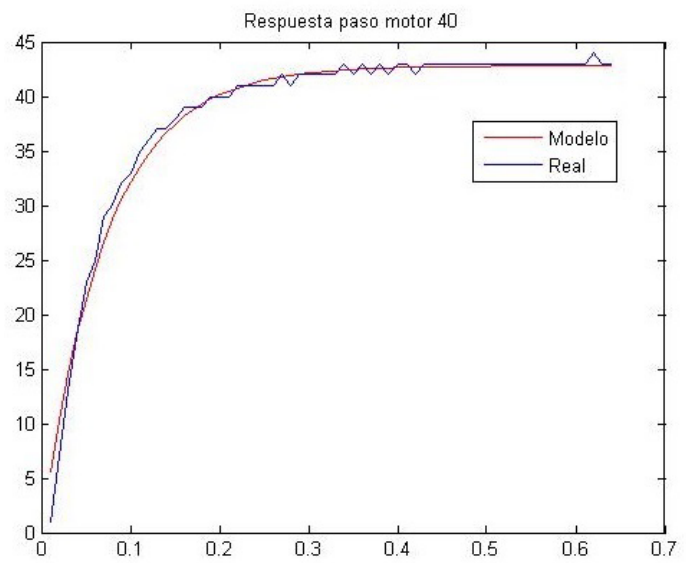

Fig. 5. Comparación entre la respuesta real y la simulada (Valderrama, 2013)

Como segunda parte del proyecto se realiza la verificación del control utilizado en la tesis de grado Valderrama (2013), en la Figura 6 se simula el control tanto en tiempo discreto como en tiempo continuo, al aplicar el controlador PI ya diseñado en la tesis de grado Valderrama (2013), teniendo en cuenta que la entrada va a variar entre $0 \%$ y $100 \%$, se simula el sistema con 
un punto de operación de 40\%. En la Figura 7 se presentan los resultados de la simulación del control continuo y discreto.

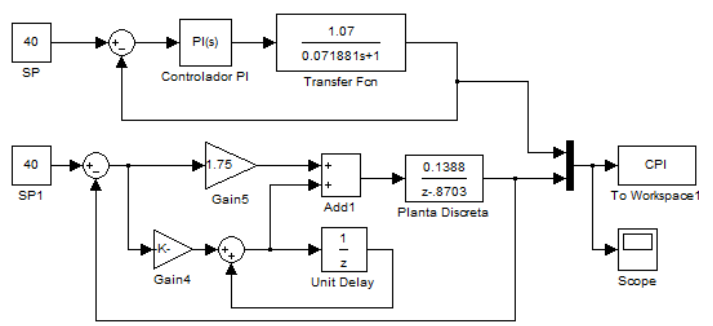

Fig. 6. Diagrama de bloques del control de cada motor en Matlab simulink®

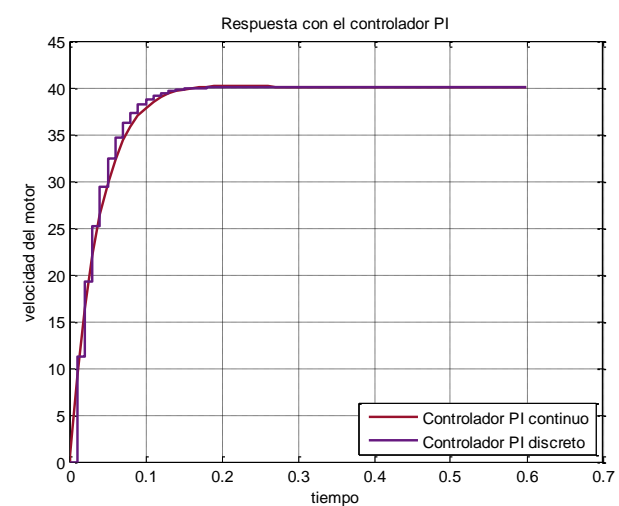

Fig. 7. Respuesta del control de velocidad del motor en tiempo continuo y discreto ante una entrada de $40 \%$

\section{METODOLOGÍA UTILIZADA EN LA SIMULACIÓN}

Teniendo en cuenta los artículos, libros consultados y la tesis de grado Valderrama (2013), y por medio del software de simulación Matlab simulink® y el Fuzzy Logic Toolbox de Matlab®, se procedió a realizar el diseño y simulación de un control alternativo que mejore la respuesta en velocidad para valores bajos y medios de respuesta de los motores anteriormente mencionados Diaz J. (2008).

Primero se realizó un diseño inicial teniendo en cuenta como entradas del controlador difuso el error y la diferencia del error, sin embargo, se encontró que el controlador genera un error en estado estacionario, por lo que se continuo con otro diseño de un controlador difuso utilizando como entrada del controlador el error y la integral del error Ogata, K. (2010).

\subsection{Diseño de controlador difuso}

Se diseño un controlador difuso teniendo en cuenta la variable lingüística; error (E) y los conjuntos difusos negativo $(\mathrm{N})$, cero (C) y positivo $(\mathrm{P})$, las funciones de pertenencia para esta variable se grafican en la Figura 8, también se designó la variable lingüística; integral del error (IE) e igualmente se definieron los conjuntos difusos negativo (N), cero (C) y positivo $(\mathrm{P})$ para la variable integral del error Contreras, J. (2010), las funciones de pertenecía se muestran en la gráfica de la Figura 10.

El diseño de las funciones de pertenencia de los conjuntos difusos se realizó haciendo un análisis de la salida que deseada del sistema en cada una de las posibles combinaciones de los conjuntos difusos de error e integral del error (pertenencias relacionadas en la salida), después de crear las respectivas reglas lógicas de implicación. También se tuvo en cuenta que el controlador escogido es tipo Mamdani con defuzzificación por medio de centroide Del Brío, B. (2006).

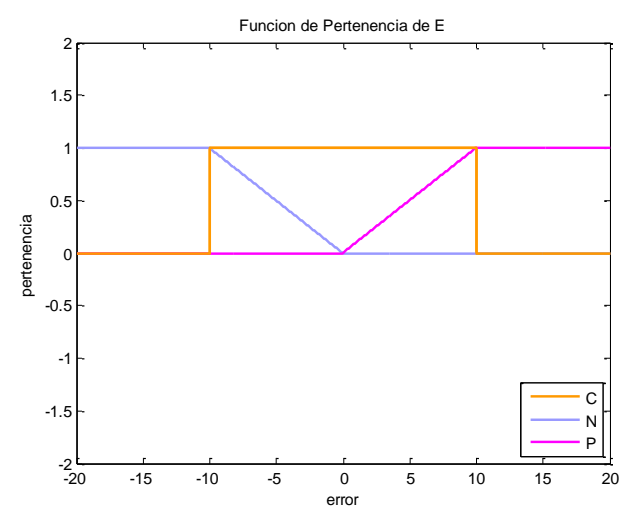

Fig. 8. Funciones de pertenencia del error negativo $(N)$, cero $(C)$ y positivo $(P)$

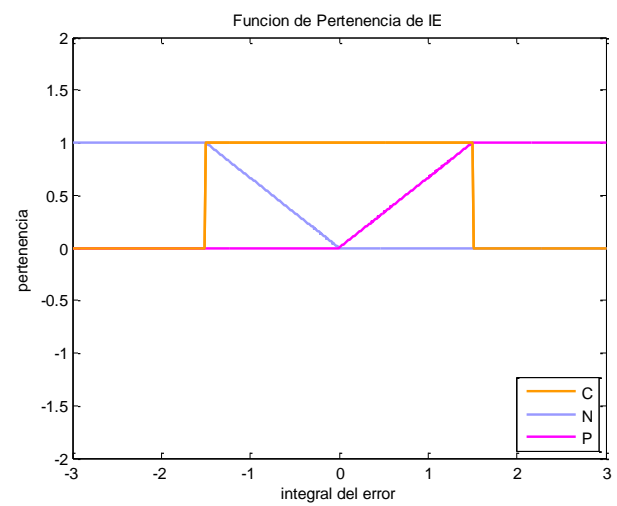

Fig. 9. Funciones de pertenencia de la integral del error negativo $(N)$, cero $(C)$ y positivo $(P)$

Luego se crea la variable lingüística de la salida (S) y se asigna los siguientes conjuntos borrosos; bajo (B), normal (N) y Alto (A). En la Figura 10 se grafican los conjuntos de pertenecía, seleccionados para que entregue valores entre un rango de $0 \%$ a $100 \%$ o $-100 \%$ a $0 \%$ en la salida de forma simétrica. 


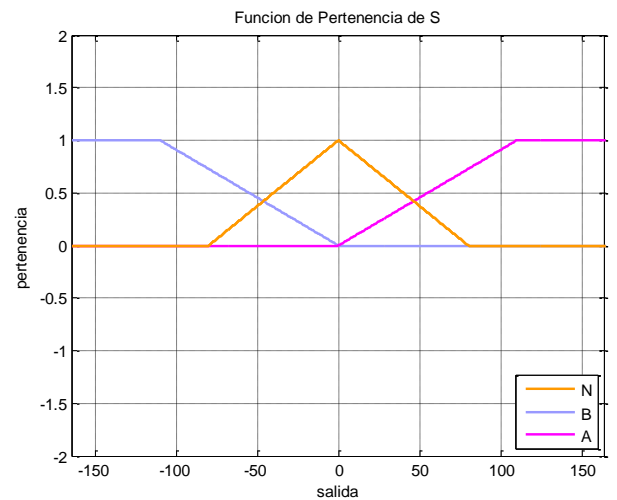

Fig. 10. Funciones de pertenencia de la salida del control difuso bajo (B), normal (N) y Alto (A)

Finalmente se crea la tabla de relaciones difusas que relacionan la entrada con la salida la cual se muestra en la Tabla 1 (Del Brío, 2006). Estas reglas de implicación indican el conjunto borroso de salida dependiendo de las posibles combinaciones de los conjuntos difusos de error e integral del error (entradas).

En la Figura 11 se grafica la superficie de salida versus el error y el error integral, aquí se verifica una convergencia de error y de la integral del error a 0 .

\section{Tabla 1: Base de reglas}

\begin{tabular}{cccc}
\hline $\mathbf{E} / \mathbf{I E}$ & $\mathbf{N}$ & $\mathbf{C}$ & $\mathbf{P}$ \\
\hline $\mathbf{N}$ & $\mathrm{B}$ & $\mathrm{B}$ & $\mathrm{N}$ \\
$\mathbf{C}$ & $\mathrm{B}$ & $\mathrm{N}$ & $\mathrm{A}$ \\
$\mathbf{P}$ & $\mathrm{N}$ & $\mathrm{A}$ & $\mathrm{A}$ \\
\hline
\end{tabular}

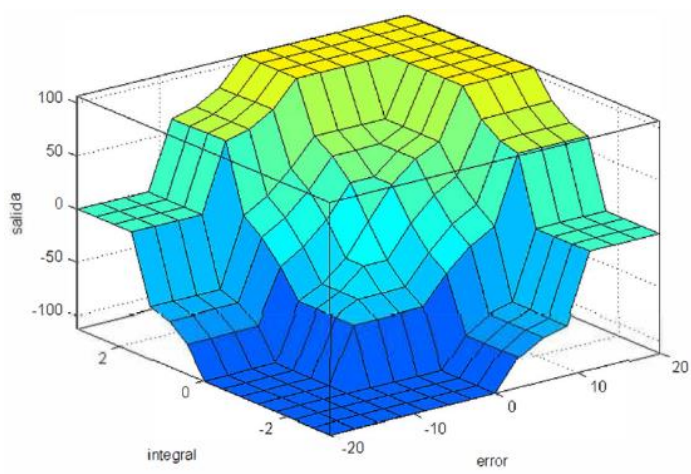

Fig. 11. Superficie de respuesta del controlador difuso de Fuzzy Logic Toolbox de Matlab®

\subsection{Simulación del controlador difuso}

Teniendo en cuenta que el sistema de control se desarrolla en un microcontrolador, se diseña, modela y simula el control difuso en tiempo discreto. En la Figura 12, se muestra el diagrama de bloques del control difuso en tiempo discreto.

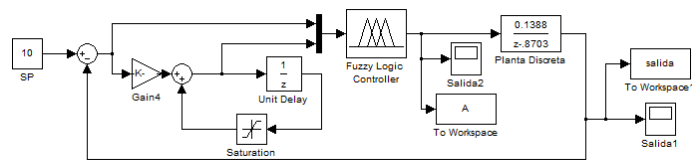

Fig. 12. Diagrama de bloques del control difuso en Matlab simulink®

Las conjuntos difusos y reglas para el bloque del control lógico difuso se diseñan con ayuda del Fuzzy Logic Toolbox de Matlab® tal como se muestra en la Figura 13.

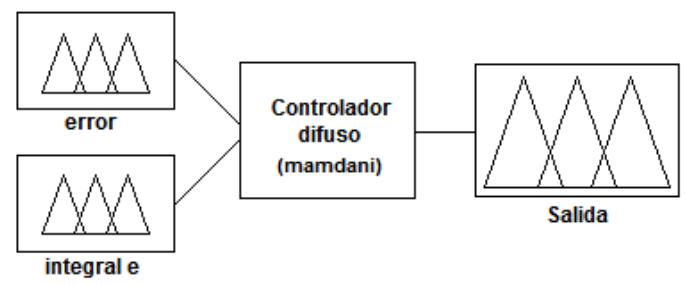

Fig. 13. Reglas del controlador difuso en el Fuzzy Logic Toolbox de Matlab®

\subsection{Comparación del controlador PI contra el controlador difuso alterno}

Para realizar la comparación entre los dos modelos se colocan los dos controladores en la misma simulación con una entrada compartida por los dos. Como lo que se quiere comparar es la salida, se ponen las dos salidas en una misma grafica de modo que se pueda realizar la comparación Galdrón, O. (2016). En la Figura 14 se muestra el diagrama de bloques que se desarrolló en Matlab simulink® para hacer la simulación y comparación.

Hay que tener en cuenta que según la tesis de grado Valderrama (2013) después del 9\% el robot comienza a moverse porque debe vencer la inercia, por tal razón se comenzó a probar con el valor inicial del $10 \%$.

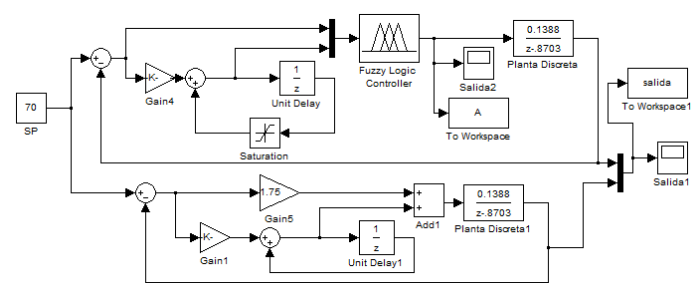

Fig. 14. Diagrama de bloques de la comparación del control PI y el control difuso en Matlab simulink $®$

\section{RESULTADOS}

Los resultados obtenidos mediante las simulaciones mostraron mejoras en la rapidez. Hay que tener en cuenta que el primer diseño que se realizo tenía error en estado estacionario porque las entradas del controlador difuso fueron 
el error y la diferencia del error, resultado similar al de Gualdrón, O. (2016). Después se diseñó un controlador difuso utilizando como entrada el error y la integral del error, obteniendo una respuesta más rápida para valores bajos y medios como se estaba buscando, estos valores en los que la respuesta difusa es más rápida son; del $10 \%$ al $60 \%$, como se observa en las Figuras 19, 20 y 21 .

\subsection{Resultados de las simulaciones}

Como simulación inicial en la grafica de la Figura 15 se tiene el resultado de la simulación del control PD difuso (tiene como entrada al controlador el error y la diferencia del error 'derivada') el cual se tuvo que cambiar porque el error en estado estacionario es muy significativo respecto al valor de referencia. Por esta razón en el artículo de Diaz J. (2008) utilizan un PWM el cual corrige el error.

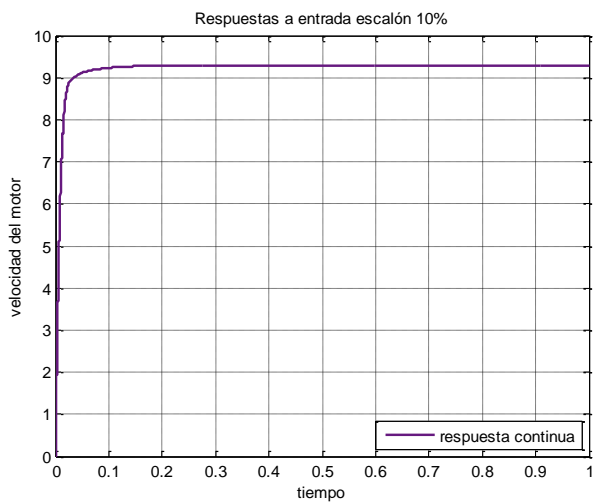

Fig. 15. Respuesta de velocidad del motor con un control PD difuso en tiempo continuo ante una entrada de $10 \%$

Para realizar el controlador se enfocó en los valores de bajo y medio porcentaje, manipulado las funciones de pertenecía de los conjuntos borrosos de entrada y salida para mejorar en este rango el tiempo de respuesta. Después de realizar varias simulaciones se encontró que el valor donde comienza a tener respuestas muy similares es alrededor del $65 \%$ como se muestra en la Figura 18, por lo que el diseño se va a centrar en el rango de valores del $10 \%$ al $60 \%$. Con estos datos se encuentra que un valor razonable para el limitador (saturation) de la parte integral es de -1.4 a 1.4. Estos valores ayudan a tener una respuesta con un sobreimpulso mínimo. Este limitador con esos valores genera error en estado estacionario para valores iguales o mayores a $70 \%$. Teniendo en cuenta lo anterior se sintonizó la función de pertenencia de la salida.

En la Figura 16 se muestra la simulación de la respuesta del control difuso para una entrada del $10 \%$ mientras que en la Figura 17 se muestra la simulación de la respuesta del control difuso para una entrada del $40 \%$.

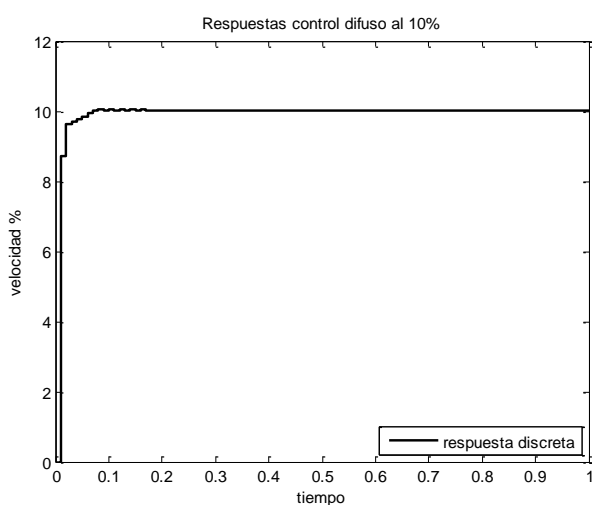

Fig. 16. Respuesta de velocidad del motor con un control PI difuso en tiempo discreto ante una entrada de $10 \%$

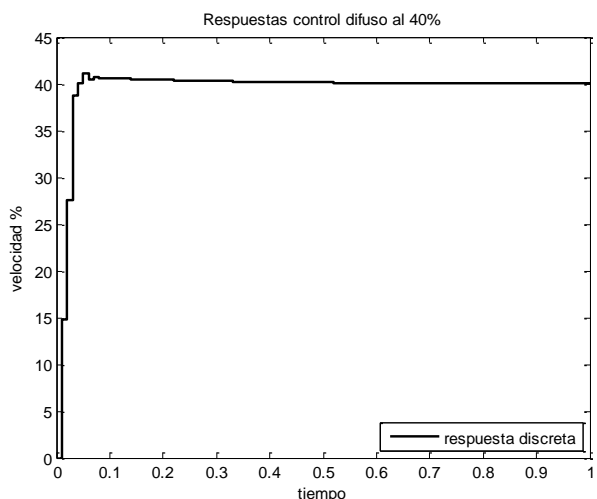

Fig. 17. Respuesta de velocidad del motor con un control PD difuso en tiempo discreto ante una entrada de $40 \%$

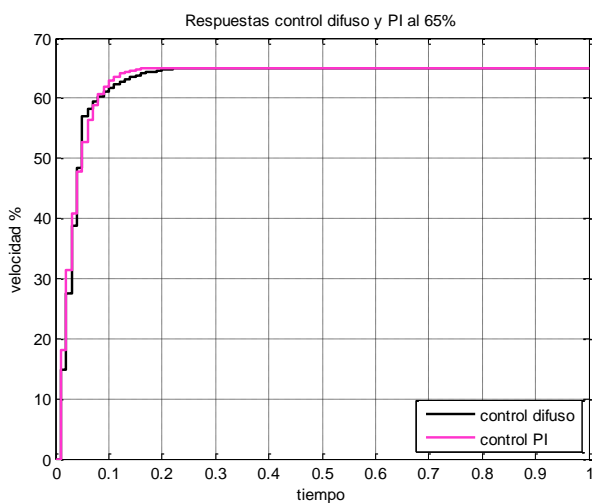

Fig. 18. Respuesta de velocidad del motor con un control PI difuso y PI clásico en tiempo discreto ante una entrada de $65 \%$

\subsection{Resultados de la comparación}

En las Figuras 19, 20 y 21 se muestra la comparación por simulación de los valores del $10 \%, 30 \%$ y $50 \%$ respectivamente, concluyendo que el control difuso tiene mejores resultados en tiempo de respuesta en simulación Diaz J. 
(2008), para valores de $10 \%$ al $60 \%$ que el PI clásico implementado en la tesis de grado Valderrama (2013), hay que tener en cuenta las limitaciones físicas del sistema y un tiempo de retado que no se tuvo en cuenta debido al tiempo de respuesta.

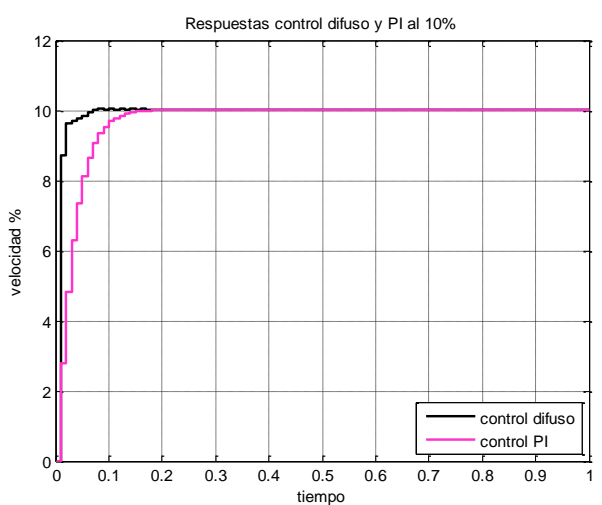

Fig. 19. Respuesta de velocidad del motor con un control PI difuso y PI clásico en tiempo discreto ante una entrada de $10 \%$

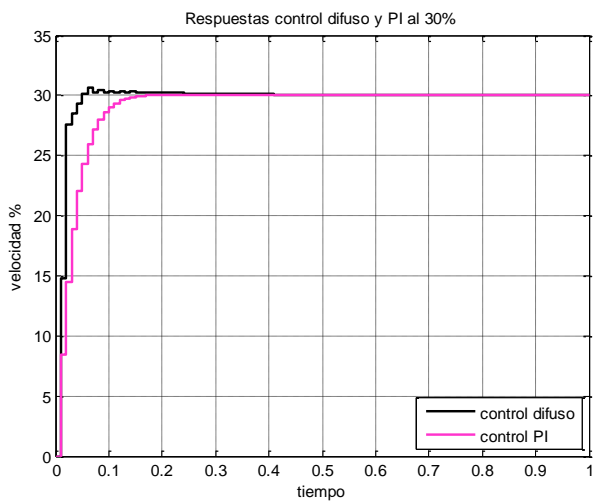

Fig. 20. Respuesta de velocidad del motor con un control PI difuso y PI clásico en tiempo discreto ante una entrada de $30 \%$

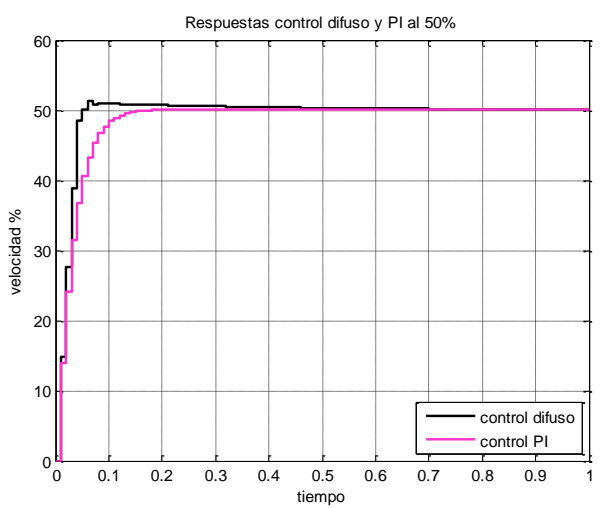

Fig. 21. Respuesta de velocidad del motor con un control PI difuso y PI clásico en tiempo discreto ante una entrada de $50 \%$

Para las tres respuestas mostradas en las Figuras 19,20 y 21 donde se comparan los dos controladores, y otras simulaciones más que no se muestran en el artículo en el rango del $10 \%$ al $60 \%$, se obtiene una mejora del $65 \%$ en el tiempo de respuesta aproximadamente Diaz J. (2008). Lo que demuestra que para esos valores se recomienda utilizar el controlador alternativo difuso, sin embargo, no se descarta del todo el control PI clásico, ya que lo que se busca es que en los rangos de $10 \%$ (valor con el cual se vence la inercia) al $60 \%$ se utilice el controlador difuso y para valores mayores al $60 \%$ se utilice el control clásico PI que ya se encuentra funcionando en la tesis de grado Valderrama (2013).

\section{CONCLUSIONES}

Se logro el diseño y simulación de un controlador difuso PI, permitiendo realizar una comparación de desempeño en tiempo de respuesta de velocidad de un sistema de primer orden contra un controlador PI clásico Diaz J. (2008).

Los resultados obtenidos inicialmente con un controlador difuso PD aplicado al sistema, daba como respuesta un error en estado estacionario no deseado, por tal motivo se cambiaron diferentes parámetros como conjuntos difusos, universo de discurso, cantidad de reglas, sin embrago no se obtuvo una respuesta mejorada de la que ya existía.

Aunque el control difuso se basa en una metodología de la incorporación de reglas heurísticas (Sala, 2009, p1), en diferentes aplicaciones se ha llegado a resultados con mejores características de desempeño. En este trabajo al implementar un controlador difuso PI se evidencia claramente que los tiempos de respuesta son $65 \%$ más eficientes en tiempo de respuesta que el controlador PI clásico que se tenía para valores del $10 \%$ al $60 \%$. No obstante, se propone el uso del PI clásico para valores del $60 \%$ al $100 \%$

Definir de forma óptima las funciones de pertenencia y el universo de discurso es el punto de partida para determinar la estructura de las reglas, y así garantizar la compensación que requiere el sistema para tener una respuesta deseada.

\section{REFERENCIAS}

Alvernia Acevedo, S., \& Rico Bautista, D. (2017). Análisis de una red en un entorno IPV6: una mirada desde las intrusiones de red y el modelo TCP/IP. REVISTA COLOMBIANA DE 
TECNOLOGÍAS DE AVANZADA, 1(29).

Ahmed, K. Y. (2016). Comparison of fuzzy logic control and PI control for a three-level rectifier based on voltage oriented control. IEEE, 1-6.

Contreras, J. (2010). Identificación y control difuso de un sistema de nivel de líquidos en dos tanques. Revista Colombiana de Ingenierias de Avanzada, 2(16), 7.

Del Brío, B. (2006). Redes neuronales y sistemas difusos. Mexico: Alfaomega.

Diaz, J. (2008). Control inteligente utilizando logica fuzzy de un motor de inducción controlado por campo orientado. Revista Colombiana de Ingenierias de Avanzada, 2(12), 7.

F. A.L. González, M. M. H. Cely. (2013). Estudio del potencial eólico y solar de Cúcuta, Norte de Santander. REVISTA COLOMBIANA DE TECNOLOGÍAS DE AVANZADA, ISSN: 1692-7257. 2(22).

Gualdrón, O. (2016). Implementación de una estrategia de control dif uso para aumentar la producción de crudo en pozo petrolero. Revista Colombiana de Ingenierias de Avanzada, 1(17), 6.

Jing, J. (2016). The fuzzy-PID control of brushless DC motor. 1-5.

Leal González, F., \& Hernandez Cely, M. (2013). Estudio del potencial eólico y solar de Cúcuta, Norte de Santander. REVISTA COLOMBIANA DE TECNOLOGÍAS DE AVANZADA, 2(22).

Li, L. (2017). Research on Air Conditioning System of Subway Station Based on Fuzzy PID Control. IEEE, 1-4.

Morales, L. (2009). Análisis y comparación entre un controlador PI difuso y un controlador PI óptimo convencional para un conversor reductor. Ingeniería e Investigación.

Noda, I. (s.f.). Robocup federation. Recuperado en Septiembre de 2018, de http://www.robocup.org/leagues/7
Ogata , K. (2010). Ingeniería de cotrol moderna (Vol. 5). Madrid: Pearson.

S. A. A. Acevedo, D. R. Bautista. (2017). Análisis de una red en un entorno IPV6: una mirada desde las intrusiones de red y el modelo TCP/IP. REVISTA COLOMBIANA DE TECNOLOGÍAS DE AVANZADA, ISSN: 1692-7257. 1(29).

Sala, A. (2009). Reduciendo distancias entre el control borroso y elcontrol no lineal: luces y sombras. Revista Iberoamericana de Automática e informática industrial , 10.

Valderrama, J. (2013). Diseño de leyes de control basadas en la teoría de la estabilidad. Bogota. Obtenido de https://repository.javeriana.edu.co/bitstr eam/handle/10554/15394/ValderramaRi nconJoaquinAndres2013.pdf?sequence $=$ 1 